\title{
Normal and Locally Normal States
}

\author{
DEREK W. ROBINSON \\ Centre Universitaire; Marseille-Luminy
}

Received July 12, 1970

\begin{abstract}
It is shown that if $\mathfrak{Q}$ is an irreducible $C^{*}$ algebra on a Hilbert space $\mathscr{H}$ and $N$ is the set of normal states of $\mathfrak{U}$ then the weak and uniform topologies on $N$ coincide and

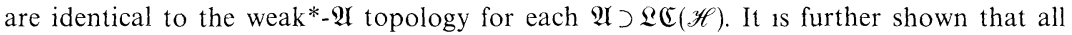
weak* topologies coincide with the uniform topology on the set of normal states with finite energy or with finite conditional entropy. A number of continuity properties of the spectra of density matrices, the mean energy, and the conditional entropy are also derived. The extension of these results to locally normal states is indicated and it is established that locally normal factor states are characterized by a doubly uniform clustering property.
\end{abstract}

\section{Introduction}

We consider subsets of normal and locally normal states over $C^{*}$ algebras which have the property that all induced weak* topologies coincide. These considerations are motivated by quantum statistical mechanics where the physically relevant structure appears to be given by subsets of locally normal states equipped with the weak* topology of the associated quasi-local algebra. As there is an inherent ambiguity in the choice of the local algebras which generate the quasi-local algebra, it is of interest to find that on most sets of physical relevance, e.g. the set of states with finite conditional entropy, all possible choices of the local algebras lead to the same weak* topology. It is also shown that the continuity properties of a number of interesting functions, such as the energy or conditional entropy, are also independent of the choice of local algebra.

\section{Normal States and Density Matrices}

Let $\mathscr{H}$ be a Hilbert space. A density matrix is defined to be a nonnegative, trace-class, operator $\varrho$ on $\mathscr{H}$ normalized such that

$$
\operatorname{Tr}_{\mathscr{H}}(\varrho)=1 .
$$


The set of density matrices can be topologized in various manners with the aid of the Hilbert space structure and for our purposes, it is convenient to consider the topologies induced by irreducible algebras of bounded operators on $\mathscr{H}$.

Let $\mathfrak{A}$ be an irreducible $C^{*}$ algebra of bounded operators on $\mathscr{H}$. We can associate with each density matrix $\varrho$ a normal state $\omega_{\varrho}$ by the definition

$$
\omega_{\varrho}(A)=\operatorname{Tr}_{\mathscr{H}}(\varrho A)
$$

and the set of density matrices is isomorphic to the set $N$ of normal states of $\mathfrak{A}$. In general $N$ is a proper convex subset of the set $E_{\mathfrak{Q}}$ of states (positive, linear, normalized, functionals) over $\mathfrak{U}$. In the following we consider the set $N$ as the central object and view this set as the common component of all the sets $E_{\mathfrak{A}}$ associated with $C^{*}$ algebras $\mathfrak{A}$ acting on $\mathscr{H}$.

The uniform topology on $N$ is a metric topology defined by the distance

$$
\left\|\omega_{\varrho}-\omega_{\sigma}\right\|=\sup _{A \in \mathscr{H},\|A\|=1}\left|\omega_{\varrho}(A)-\omega_{\sigma}(A)\right| .
$$

Next recall that the trace norm $\||A \||$ of an operator $A$ on $\mathscr{H}$ is given by

$$
\left\||| A\left|\|=\sup _{X \in \mathscr{Q}(\mathscr{H}),\|X\|=1}\right| \operatorname{Tr}_{\mathscr{H}}(X A) \mid .\right.
$$

The Kaplansky density theorem however allows us to deduce that the supremum in this definition can be taken over any irreducible sub $C^{*}$ algebra $\mathfrak{U}$ of $\mathfrak{Q}(\mathscr{H})$. Hence we deduce that

$$
\left\|\omega_{\varrho}-\omega_{\sigma}\right\|=\|\left\lfloor-\sigma \mid \|=\operatorname{Tr}_{\mathscr{H}}(|\varrho-\sigma|)\right.
$$

and the uniform topology on $N$ is independent of the particular algebra $\mathfrak{A}$ used in its definition; the uniform topology on $N$ coincides with the trace-norm topology on the density matrices. A basis of neighbourhoods for the uniform topology is given by

$$
\mathscr{U}\left(\omega_{\varrho} ; \varepsilon\right)=\left\{\omega_{\sigma} ; \omega_{\sigma} \in N,\left\|\omega_{\varrho}-\omega_{\sigma}\right\|<\varepsilon\right\}
$$

where $\omega_{\varrho} \in N$ and $\varepsilon>0$. It is readily checked that $N$ is uniformly complete.

Next for each irreducible $C^{*}$ algebra $\mathfrak{A}$ on $\mathscr{H}$ we define a weak*- $\mathfrak{A}$ topology by the following basis of neighbourhoods

$$
\mathscr{W}_{\mathscr{Q}}\left(\omega_{\varrho} ; A_{1}, \ldots, A_{n}, \varepsilon\right)=\left\{\omega_{\sigma} ; \omega_{\sigma} \in N,\left|\omega_{\sigma}\left(A_{i}\right)-\omega_{\varrho}\left(A_{i}\right)\right|<\varepsilon i=1, \ldots, n\right\}
$$

where $\omega_{\varrho} \in N, A_{1} \in \mathfrak{U}, \ldots, A_{n} \in \mathfrak{U}$, and $\varepsilon>0$. The weak*-2l topology is coarser than the uniform topology. Further each weak*-2I topology is coarser than the weak*- $\mathfrak{Q}(\mathscr{H})$ topology. We refer to this last topology as the weak topology on $N$ because for each $\mathfrak{A}$ it coincides with the weak, or $\sigma\left(\mathfrak{A}^{*}, \mathfrak{A}^{* *}\right)$, topology restricted to $N$. (This follows from the irreducibility of $\mathfrak{A}$ and the identification of its bidual $\mathfrak{A}^{* *}$ with the 
von Neumann enveloping algebra of $\mathfrak{A}$.) Note that if $\mathfrak{A}_{1} \subset \mathfrak{A}_{2}$ then the

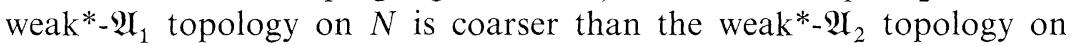
$N$ but in general the weak* topologies are not comparable. Note that if $\mathscr{H}$ is infinite-dimensional then the set $N$ of normal states is not closed in any of the weak* topologies but is a relatively compact subset of the dual of $\mathfrak{A}$. If $\mathfrak{C}$ is the $C^{*}$ algebra of compact operators on $\mathscr{H}$ then $N=E_{\mathbb{C}}$.

In the case that $\mathscr{H}$ is finite-dimensional all the above topologies of course coincide. We next prove a result of this nature for the case that $\mathscr{H}$ is infinite-dimensional.

Theorem 1. Let $\mathbb{C}$ be the $C^{*}$ algebra of compact operators on the Hilbert space $\mathscr{H}$ and $\mathfrak{A}$ a $C^{*}$ algebra on $\mathscr{H}$ with the property that $\mathfrak{A} \supset \mathfrak{C}$. Let $N$ denote the set of normal states.

The weak ${ }^{*}-\mathfrak{H}$, weak, and uniform, topologies coincide on $N$.

Proof. Let $\omega_{e}$ be an arbitrary state in $N$. We will show that each neighbourhood of $\omega_{\varrho}$ in the basis defining the uniform topology on $E_{\mathscr{Q}}$ contains a neighbourhood of the basis which defines the weak*-2 topology. The desired result then follows from the ordering of the topologies.

Now $\omega_{\varrho}$ is normal and hence we can choose for each $\varepsilon>0$ a finite rank projector $E_{\varrho}$ on $\mathscr{H}$ such that

$$
0 \leqq \omega_{\varrho}\left(1-E_{\varrho}\right)<\varepsilon .
$$

For example $E_{\varrho}$ could be chosen to be a suitable spectral projector of the density matrix $\varrho$ associated with $\omega_{\varrho}$. Now we can define a weak*- $\mathfrak{U}$ neighbourhood of $\omega_{\varrho}$ by

$$
\mathscr{W}_{\mathfrak{I I}}\left(\omega_{\varrho} ; \varepsilon\right)=\left\{\omega ; \omega \in E_{\mathfrak{Q}}, \sup _{A \in \mathfrak{R},\|A\|=1}\left|\omega\left(E_{\varrho} A E_{\varrho}\right)-\omega_{\varrho}\left(E_{\varrho} A E_{\varrho}\right)\right|<\varepsilon\right\} .
$$

In this form it is not immediately transparent that $\mathscr{W}_{\mathscr{P l}}\left(\omega_{\varrho} ; \varepsilon\right)$ is a weak ${ }^{*} \mathfrak{A}$ neighbourhood but this is a consequence of the finite rank of $E_{\varrho}$. Now for each $\omega \in \mathscr{W}_{\mathfrak{Q}}\left(\omega_{\underline{Q}} ; \varepsilon\right)$ we have

$$
\omega\left(1-E_{\varrho}\right)=\omega_{\varrho}\left(1-E_{\varrho}\right)+\omega_{\varrho}\left(E_{\varrho}\right)-\omega\left(E_{\varrho}\right)
$$

and hence

$$
\begin{aligned}
0 \leqq \omega\left(1-E_{\varrho}\right) & \leqq\left|\omega_{\varrho}\left(1-E_{\varrho}\right)\right|+\left|\omega_{\varrho}\left(E_{\varrho}\right)-\omega\left(E_{\varrho}\right)\right| \\
& <2 \varepsilon .
\end{aligned}
$$

Further for each $A \in \mathfrak{A}$ we can apply the triangle inequality and Schwartz inequality to find

$$
\begin{aligned}
\left|\omega(A)-\omega_{\varrho}(A)\right| \leqq & \left|\omega\left(E_{\varrho} A E_{\varrho}\right)-\omega_{\varrho}\left(E_{\varrho} A E_{\varrho}\right)\right| \\
& +2\|A\|\left(\sqrt{\omega\left(1-E_{\varrho}\right)}+\sqrt{\left.\omega_{\varrho}\left(1-E_{\varrho}\right)\right)}\right.
\end{aligned}
$$


and hence for $\omega \in \mathscr{W}_{\mathfrak{I}}(\varrho ; \varepsilon)$ one concludes that

$$
\sup _{A \in \mathfrak{Q}\|A\|=1}\left|\omega(A)-\omega_{\varrho}(A)\right|<\varepsilon+2 \sqrt{\varepsilon}+2 \sqrt{2 \varepsilon},
$$

i.e. the weak ${ }^{*}-\mathfrak{U}$ neighbourhood $\mathscr{W}_{\mathfrak{I}}\left(\omega_{\varrho} ; \varepsilon\right)$ is contained in the uniform neighbourhood $\mathscr{U}\left(\omega_{\varrho} ; \delta\right)$ where

$$
\mathscr{U}\left(\omega_{\varrho} ; \delta\right)=\left\{\omega ; \omega \in E_{\mathfrak{U}},\left\|\omega-\omega_{\varrho}\right\|<\delta\right\}
$$

and

$$
\delta=\varepsilon+2 \sqrt{\varepsilon}+2 \sqrt{2 \varepsilon} .
$$

Thus the proof of the theorem is complete.

It is natural to ask whether the conditions of the theorem imply that all weak*-2 topologies coincide on $N$ even if $\mathfrak{A} \supset \mathfrak{C}$ but one can show by example that this is not the case.

The above result can be restated in terms of Hilbert space topologies on subsets of the set of density matrices, or as a statement concerning convergence properties. Note that as each density matrix is a compact operator it is natural to consider in the latter connection the convergence properties of eigenvalues and eigenprojectors. If $A$ is a non-negative compact operator on $\mathscr{H}$ we denote by $\left(\lambda_{m}(A)\right)_{m \geqq 1}$ the eigenvalues of $A$ arranged in decreasing order and repeated according to multiplicity. We also associate with $A$ the spectral decomposition

$$
A=\int_{0}^{\infty} d E_{A}(\lambda) \lambda
$$

and for each open subset $\Delta$ of $[0, \infty)$ we define the spectral projector

$$
E_{A}(\Delta)=\int_{\Delta} d E_{A}(\lambda)
$$

Theorem 2. Let $\mathfrak{U}$ be a $C^{*}$ algebra of bounded operators on the Hilbert space $\mathscr{H}$ which contains the $C^{*}$ algebra $\mathbb{C}$ of compact operators on $\mathscr{H}$. Let $\left(\varrho_{\alpha}\right)$ be a directed set of density matrices and $\left(\omega_{\varrho_{\alpha}}\right)$ the corresponding directed set of normal states over $\mathfrak{A}$.

The following conditions are equivalent

1. $\left(\omega_{\varrho_{\alpha}}\right)$ converges in the uniform topology to $\omega_{\varrho}$.

2. $\left(\omega_{\varrho_{\alpha}}\right)$ converges in the weak*- $\mathfrak{U}$ topology to the normal state $\omega_{\varrho}$.

3. $\left(\varrho_{\alpha}\right)$ converges to $\varrho$ in the trace-norm topology.

4. $\left(\varrho_{\alpha}\right)$ converges to the density matrix $\varrho$ in the weak Hilbert space topology.

5. $\left(\varrho_{\alpha}\right)$ converges to the density matrix $\varrho$ in the sense that the eigenvalue $\lambda_{m}\left(\varrho_{\alpha}\right)$ converges to $\lambda_{m}(\varrho)$ for $m=1,2, \ldots$ and the eigenprojector $E_{\varrho_{\alpha}}(\Delta)$ converges uniformly to $E_{\varrho}(\Delta)$ for each interval $\Delta=\left(\delta_{1}, \delta_{2}\right)$ such that the extremities do not belong to the spectrum of $\varrho$. 
The eigenvalues $\left(\lambda_{m}\left(\varrho_{\alpha}\right)\right)_{m \geqq 1}$ and $\left(\lambda_{m}(\varrho)\right)_{m \geqq 1}$ of $\left(\varrho_{\alpha}\right)$ and $\varrho$ satisfy the inequality

$$
\sum_{m \geqq 1}\left|\lambda_{m}\left(\varrho_{\alpha}\right)-\lambda_{m}(\varrho)\right| \leqq\left\|\varrho_{\alpha}-\varrho\right\| \mid
$$

Proof. Note that in condition 2 the specification that the limit element is a normal state is essential, as is the specification in 4 and 5 that the limit element is a density matrix. If similar specifications are made all other intermediate forms of Hilbert space convergence, ultra-weak, strong, ultra-strong, and uniform, are also of course equivalent to the conditions of the theorem.

We remark that the estimate for the eigenvalues is true for any traceclass operators $\varrho_{\alpha}$ and $\varrho$; a proof is given, for example, in the appendix of [2].

The equivalence of 1 and 2 is a result of Theorem 1 ; the equivalence of 1 and 3 is discussed at the beginning of the section; the equivalence of 3 and 4 is actually established in the proof of Theorem 1. It is known that condition 3 implies condition 5; see, for example, [3] pages 365-367, where a more general result is established. The proof of the theorem can be completed by showing that 5 implies 4; this is essentially trivial and we will omit the details of a formal proof.

Note that in [3] one actually establishes that if $\alpha$ is large enough, and consequently $\left\|\varrho-\varrho_{\alpha}\right\| \mid$ is small enough, then

$$
\left\|E_{\varrho_{\alpha}}(\Delta)-E_{\varrho}(\Delta)\right\|<C(\Delta)\left\|\varrho-\varrho_{\alpha}\right\|
$$

where $C(\Delta)$ is positive and independent of $\alpha$.

One can restate the partial content of the above theorem as follows.

Corollary 1. Let $\left(A_{\alpha}\right)$ be a directed set of non-negative trace-class operators on $\mathscr{H}$. The following conditions are equivalent.

1. $\left(A_{\alpha}\right)$ converges in the weak operator topology to a trace-class operator $A$ and $\operatorname{Tr}_{\mathscr{H}}\left(A_{\alpha}\right)$ converges to $\operatorname{Tr}_{\mathscr{H}}(A)$.

2. $\left(A_{\alpha}\right)$ converges in the trace-norm topology to $A$.

This corollary is reminiscent of the well-known fact that a sequence $\Psi_{n}$ of vectors in $\mathscr{H}$ converges strongly to $\Psi$ if, and only if, $\Psi_{n}$ converges weakly to $\Psi$ and $\left\|\Psi_{n}\right\|$ converges to $\|\Psi\|$.

\section{States of Bounded Energy}

In this section we consider a $C^{*}$ algebra $\mathfrak{A}$ acting irreducibly on $\mathscr{H}$ and special subsets of the normal states over $\mathfrak{A}$. These subsets will be singled out by domain requirements for an unbounded operator which has the properties typical of a local Hamiltonian in quantum statistical 
mechanics; physically the subsets correspond to the states of finite energy.

Let $H$ be a lower semi-bounded self-adjoint operator on $\mathscr{H}$ with the property that the resolvent of $H$ is compact, i.e. $H$ has discrete spectrum with finite multiplicities for each of the eigenvalues. We first define an extension of each normal state to the operator $H$. There are various equivalent ways of doing this but the following is most economical for our present purposes.

Introduce the spectral representation of $H$ by

$$
H=\int_{-\infty}^{\infty} d E_{H}(\lambda) \lambda
$$

then for each $m \in[0, \infty)$, such that $m$ is not in the spectrum of $H$, define $H_{m} \in \mathfrak{Q}(\mathscr{H})$ by

$$
H_{m}=\int_{\lambda \leqq m} d E_{H}(\lambda) \lambda .
$$

Now for each $\omega_{\varrho} \in N$ we define $\hat{H}(\varrho) \in[0,+\infty]$ by

$$
\hat{H}(\varrho)=\sup _{m} \omega_{\varrho}\left(H_{m}\right) .
$$

Note that if $\left(\lambda_{m}(\varrho)\right)_{m \geqq 1}$ are the eigenvalues of $\varrho$ and $\left(\Psi_{m}\right)_{m \geqq 1}$ are a corresponding complete orthonormal set of eigenfunctions then we have

$$
\hat{H}(\varrho)=\sum_{m \geqq 1} \lambda_{m}(\varrho)\left\|H^{\frac{1}{2}} \Psi_{m}\right\|^{2}
$$

where $\left\|H^{\frac{1}{2}} \Psi_{m}\right\|$ is set equal to plus infinity if $\Psi_{m}$ is not in the domain of $H^{\frac{1}{2}}$. Using a similar convention one can also make the identifications

$$
\hat{H}(\varrho)=\operatorname{Tr}_{\mathscr{H}}\left(\varrho^{\frac{1}{2}} H \varrho^{\frac{1}{2}}\right)=\operatorname{Tr}_{\mathscr{H}}\left(H^{\frac{1}{2}} \varrho H^{\frac{1}{2}}\right) .
$$

It follows from the last characterization that the function $\omega_{\varrho} \in N$ $\rightarrow \hat{H}(\varrho) \in[0,+\infty]$ is affine.

Theorem 3. Let $\mathfrak{A}$ be an irreducible $C^{*}$ algebra on the Hilbert space $\mathscr{H}$ and let $N$ denote the set of normal states. Let $H$ be a lower semi-bounded self-adjoint operator on $\mathscr{H}$ with compact resolvent and introduce the sets $D_{n}(H)$ by

$$
D_{n}(H)=\left\{\omega_{\varrho} ; \omega_{\varrho} \in N, \hat{H}(\varrho) \leqq n\right\} \quad n \geqq 0 .
$$

It follows that

1. $D_{n}(H)$ is convex and closed in the weak ${ }^{*}-\mathfrak{A}$ topology on $E_{\mathfrak{H}}$.

2. The weak ${ }^{*}-\mathfrak{2}$, weak, and uniform, topologies coincide on $D_{n}(H)$.

Proof. It clearly suffices to consider the case that $H$ is non-negative. Using the notation introduced above we define the spectral projector 
$E_{m}$ of $H$ by

$$
E_{m}=\int_{0}^{m} d E_{H}(\lambda)
$$

and note that $E_{m}$ is of finite rank if $m<+\infty$. Now for each $\omega_{\varrho} \in D_{n}(H)$ we have the inequality

$$
n \geqq \hat{H}(\varrho) \geqq \sup _{M} \omega_{\varrho}\left(\int_{m}^{M} d E_{I I}(\lambda) \lambda\right) \geqq m \omega_{\varrho}\left(1-E_{m}\right)
$$

i.e. we have

$$
0 \leqq \omega_{\varrho}\left(1-E_{m}\right) \leqq n / m
$$

uniformly for all $\omega_{0} \in D_{n}(H)^{1}$.

Next note that if $\omega_{\varrho_{\alpha}}$ is a directed set of states in $D_{n}(H)$ which converges in the weak topology of $\mathfrak{A}$, i.e. in the $\sigma\left(\mathfrak{I}^{*}, \mathfrak{I}^{* *}\right)$ topology, to a state $\omega \in E_{\mathfrak{I}}$ then one has

$$
\omega\left(E_{m}\right) \geqq 1-n / m
$$

where $E_{m}$ is identified as an element of the bidual of $\mathfrak{A}$ and $\omega$ is identified with its ultra-weakly continuous extension. It follows immediately that $\omega \in N$. Now note that as $\omega_{\varrho} \in N \rightarrow H(\varrho)$ is affine $D_{n}(H)$ is convex. But as $\hat{H}$ is defined over $N$ as the supremum of a family of weakly continuous functions it follows that it is lower semi-continuous in the weak topology on $N$. Consequently $D_{n}(H)$ is closed in the weak topology on $N$ and thus closed in the weak topology on $E_{\mathfrak{l}}$ by the argument given at the beginning of the paragraph.

It follows from Theorem 1 that the weak and uniform topologies coincide on $D_{n}(H)$ because $D_{n}(H) \subset N$ and we next demonstrate that they coincide with the weak ${ }^{*} \mathfrak{A}$ topology.

Using the above estimate and repeating the calculation used in proof of Theorem 1 we find

$$
|| \omega_{\varrho}(B)-\omega_{\sigma}(B)|-| \omega_{\varrho}\left(E_{m} B E_{m}\right)-\omega_{\sigma}\left(E_{m} B E_{m}\right) \mid \leqq 4(n / m)^{\frac{1}{2}}\|B\|
$$

for all $\omega_{\varrho}, \omega_{\sigma} \in D_{n}(H)$ and all $B \in \mathfrak{Q}(\mathscr{H})$. Now take $\omega_{\varrho} \in D_{n}(H)$ and consider the weak neighbourhood $\mathscr{W}$ of $\omega_{\varrho}$ given by

$$
\mathscr{W}\left(\omega_{\varrho}, B_{1}, \ldots, B_{p}, \varepsilon\right)=\left\{\omega_{\sigma} ; \omega_{\sigma} \in D_{n}(H),\left|\omega_{\varrho}\left(B_{i}\right)-\omega_{\sigma}\left(B_{i}\right)\right|<\varepsilon, i=1, \ldots, p\right\}
$$

where $B_{i} \in \mathbb{I}(\mathscr{H})$ with $\left\|B_{i}\right\| \leqq 1$ for $i=1, \ldots, p$, and $\varepsilon>0$. Now choose $m$ such that $0<4(n / m)^{\frac{1}{2}}<\varepsilon / 5$ and choose $A_{i} \in \mathfrak{A}$ such that

$$
\left\|\left(A_{i}-B_{i}\right) E_{m}\right\|<\varepsilon / 5, \quad\left\|A_{i}\right\| \leqq 1, \quad i=1, \ldots, p .
$$

${ }^{1}$ We are indebted to O. E. Lanford for pointing out an estimation of this nature in a related context. 
The last choice is made possible by the finite rank of $E_{m}$, the irreducibility of $\mathfrak{A}$ on $\mathscr{H}$, and the Kaplansky density theorem. Finally define the weak*-Ml neighbourhood by

$$
\begin{aligned}
& \mathscr{W}_{\mathscr{N}}\left(\omega_{\varrho} ; A_{1}, \ldots, A_{p}, \varepsilon / 5\right) \\
& \quad=\left\{\omega_{\sigma} ; \omega_{\sigma} \in D_{n}(H),\left|\omega_{\sigma}\left(A_{i}\right)-\omega_{\varrho}\left(A_{i}\right)\right|<\varepsilon / 5, i=1, \ldots, p\right\} .
\end{aligned}
$$

Using these choices and the foregoing inequality we then find for each $\omega_{\sigma} \in \mathscr{W}_{\mathscr{P}}$ that

$$
\begin{aligned}
\left|\omega_{\varrho}\left(B_{i}\right)-\omega_{\sigma}\left(B_{i}\right)\right| & <\varepsilon / 5+\left|\omega_{\varrho}\left(E_{m} B_{i} E_{m}\right)-\omega_{\sigma}\left(E_{m} B_{l} E_{m}\right)\right| \\
& <3 \varepsilon / 5+\left|\omega_{\varrho}\left(E_{m} A_{i} E_{m}\right)-\omega_{\sigma}\left(E_{m} A_{i} E_{m}\right)\right| \\
& <4 \varepsilon / 5+\left|\omega_{\varrho}\left(A_{i}\right)-\omega_{\sigma}\left(A_{i}\right)\right| \\
& <\varepsilon,
\end{aligned}
$$

i.e. we have $\mathscr{W}_{2 \mathbb{P}} \subset \mathscr{W}$ and hence the topologies coincide on $D_{n}(H)$.

Finally we prove that $D_{n}(H)$ is closed in the weak*-2 topology on $E_{\mathscr{Q}}$. Let $\omega_{\varrho_{\alpha}}$ be a directed set of states in $D_{n}(H)$ which converges in the weak*-2 topology to a state $\omega$. Thus $\left(\omega_{\varrho_{\alpha}}\right)$ is a Cauchy system for the weak*-2 topology. But the above estimates immediately show that $\left(\omega_{Q_{\alpha}}\right)$ is a Cauchy system for the uniform topology. However $N$ is complete and $D_{n}(H)$ is closed in this latter topology. Hence $D_{n}(H)$ is uniformly complete and $\left(\omega_{Q_{\alpha}}\right)$ has a uniform limit point $\omega^{\prime}$ in $D_{n}(H)$. But we must then have $\omega^{\prime}=\omega$, i.e. $\omega \in D_{n}(H)$, and so $D_{n}(H)$ is closed in the weak*-2t topology.

In physical applications $\hat{H}(\varrho)$ is interpretable as the mean value of the energy of the state $\omega_{\varrho}$. It is often useful to have an affine extension of $\hat{H}$ to the complete state space $E_{\mathscr{Q}}$ of $\mathfrak{A}$.

Corollary 2. Let $\mathfrak{A}$ be an irreducible $C^{*}$ algebra on $\mathscr{H}$. Define $\hat{H}$ as a function over the state space $E_{\mathfrak{Q}}$ of $\mathfrak{I}$ by

and

$$
\hat{H}\left(\omega_{\varrho}\right)=\hat{H}(\varrho) \quad \text { for } \quad \omega_{\varrho} \in N
$$

$$
\hat{H}(\omega)=+\infty \quad \text { for } \quad \omega \in E_{\mathfrak{Q}} \backslash N .
$$

It follows that $\omega \in E_{\mathscr{Q}} \rightarrow \hat{H}(\omega) \in[0,+\infty]$ is affine and lower semi-continuous in the weak ${ }^{*}-\mathfrak{U}$ topology.

The affinity follows because $\hat{H}$ is affine over $N$ and if $\omega^{\prime}>\omega \in E_{\mathscr{Y}} \backslash N$ then $\omega^{\prime} \in E_{\mathfrak{Q}} \backslash N$. The function is lower semi-continuous because the sets

$$
\{\omega ; \hat{H}(\omega) \leqq n\} \quad n \geqq 0
$$

are closed in the weak*-2 topology by Theorem 3 . 


\section{The Conditional Entropy}

We can assign an entropy $S(\varrho)$ to each density matrix $\varrho$ by the definition

$$
S(\varrho)=-\operatorname{Tr}_{\mathscr{H}}(\varrho \log \varrho)
$$

if $-\varrho \log \varrho$ is of trace-class and

$$
S(\varrho)=+\infty
$$

if $-\varrho \log \varrho$ is not of trace-class. Note that as $0 \leqq \varrho \leqq 1$, the operator $-\varrho \log \varrho$ is bounded and compact (we follow the usual convention of setting $-t \log t=0$ for $t=0$ ) and hence the above definition is unambiguous and defines a function $\omega_{\varrho} \in N \rightarrow S\left(\omega_{\varrho}\right)=S(\varrho)$ over the normal states.

In equilibrium statistical mechanics a function of central importance is the conditional entropy $S-\beta \hat{H}$ where $\beta$ is a positive real number which is interpreted as the inverse temperature and $\hat{H}$ is the function, introduced in the previous section, which corresponds to the mean energy. The definition of $S-\beta \hat{H}$ is however ambiguous because both $S$ and $\hat{H}$ can take the value plus infinity. In order to give an unambiguous definition of this function, one must first investigate the relations between the subsets of normal states for which $S(\varrho)=+\infty$ and $\hat{H}(\varrho)=+\infty$ respectively. We next give a result of this nature for a slightly specialized class of $H$.

Theorem $\mathbf{4}^{2}$. Let $\mathscr{B}$ denote the set of self-adjoint operators on $\mathscr{H}$ with the property that

$$
\operatorname{Tr}_{\mathscr{H}}\left(e^{-\beta H}\right)<+\infty
$$

for all $\beta>0$. For $H \in \mathscr{B}$ define $D(H)$ by

$$
D(H)=\left\{\omega_{\varrho} ; \omega_{\varrho} \in N, \hat{H}(\varrho)<+\infty\right\} .
$$

It follows that if $\omega_{\varrho} \in D(H)$ then

$$
0 \leqq-\operatorname{Tr}_{\mathscr{H}}(\varrho \log \varrho) \leqq \beta \hat{H}(\varrho)+\log \operatorname{Tr}_{\mathscr{H}}\left(e^{-\beta H}\right)<+\infty
$$

for all $\beta>0$.

Proof. Note that if $H \in \mathscr{B}$ then $H$ is automatically lower semi-bounded with compact resolvent.

Let $\left(\hat{\lambda}_{m}(\varrho)\right)_{m \geqq 1}$ be the eigenvalues of $\varrho$. The above definition of $S(\varrho)$ is equivalent to the definition

$$
S(\varrho)=\sum_{m \geqq 1}-\lambda_{m}(\varrho) \log \lambda_{m}(\varrho) .
$$

2 This theorem establishes that the set of states with finite entropy contains the unıon, over $H \in \mathscr{B}$, of the sets $D(H)$; actually the containment can be proved to be an equality. 
(As all terms in the sum are non-negative it can in all cases be unambiguously defined and the sum is finite if, and only if, $-\varrho \log \varrho$ is of traceclass.)

Next let $\left(\Psi_{m}\right)_{m \geqq 1}$ be a complete set of orthonormal eigenfunctions of $\varrho$ corresponding to the eigenvalues $\left(\lambda_{m}(\varrho)\right)_{m \geqq 1}$. We have

$$
\hat{H}(\varrho)=\sum_{m \geqq 1} \lambda_{m}(\varrho)\left\|H^{\frac{1}{2}} \Psi_{m}\right\|^{2}
$$

and using the convexity of the exponential function one finds that

$$
-\beta\left\|H^{\frac{1}{2}} \Psi_{m}\right\|^{2} \leqq \log \left(\Psi_{m}, e^{-\beta H} \Psi_{m}\right), \quad \beta>0 .
$$

Thus we have

$$
\begin{aligned}
S(\varrho)-\beta \hat{H}(\varrho) & -\log \operatorname{Tr}_{\mathscr{H}}\left(e^{-\beta H}\right) \\
& \leqq-\sum_{m \geqq 1} \lambda_{m}(\varrho)\left[\log \lambda_{m}(\varrho)-\log \frac{\left(\Psi_{m}, e^{-\beta H} \Psi_{m}\right)}{\operatorname{Tr}_{\mathscr{H}}\left(e^{-\beta H}\right)}\right] .
\end{aligned}
$$

But using the convexity inequality

$$
-x(\log x-\log y) \leqq y-x \quad x \geqq 0, y>0
$$

one concludes that

$$
\begin{aligned}
S(\varrho)-\beta \hat{H}(\varrho)-\log \operatorname{Tr}_{\mathscr{H}}\left(e^{-\beta H}\right) & \leqq-\sum_{m \geqq 1}\left[\lambda_{m}(\varrho)-\frac{\left(\Psi_{m}, e^{-\beta H} \Psi_{m}\right)}{\operatorname{Tr}_{\mathscr{H}}\left(e^{-\beta H}\right)}\right] \\
& =0 .
\end{aligned}
$$

Thus

$$
0 \leqq S(\varrho) \leqq \beta \hat{H}(\varrho)+\log \operatorname{Tr}_{\mathscr{H}}\left(e^{-\beta H}\right)<+\infty
$$

and the proof is complete.

The above results indicate the following definition for the conditional entropy.

Let $\mathfrak{A}$ be an irreducible $C^{*}$ algebra on the Hilbert space $\mathscr{H}$. For each $H \in \mathscr{B}$ and $\beta>0$ the conditional entropy is defined as a function over the state space $E_{\mathfrak{U}}$ of $\mathfrak{A}$ by

and

$$
S\left(\omega_{\varrho} ; \beta H\right)=S(\varrho)-\beta \hat{H}(\varrho) \quad \text { for } \quad \omega_{\varrho} \in D(H)
$$

$$
S(\omega ; \beta H)=-\infty \quad \text { for } \quad \omega \in E_{\mathfrak{U} \backslash} \backslash D(H) .
$$

Theorem 5. Retain the notation introduced above. For each $H \in \mathscr{B}$ and $\beta>0$ the conditional entropy is upper semi-continuous in the weak ${ }^{*}-\mathfrak{I}$ topology. The weak*-2l topology and the uniform topology coincide on the weak*-II closed subsets $D_{n}(S ; \beta H)$ defined by

$$
D_{n}(S ; \beta H)=\left\{\omega ; \omega \in E_{\mathscr{R}}, S(\omega ; \beta H) \geqq n\right\} .
$$


Proof. We first show that for each $n$ there is an $m$ such that

$$
D_{n}(S ; \beta H) \subset D_{m}(H)
$$

and hence conclude that the topologies coincide on $D_{n}(S ; \beta H)$ as a corollary of Theorem 3. If $0<\beta_{0}<\beta$ we have from Theorem 4 that

$$
\begin{aligned}
S\left(\omega_{\varrho} ; \beta H\right) & =S\left(\omega_{\varrho} ; \beta_{0} H\right)-\left(\beta-\beta_{0}\right) \hat{H}(\varrho) \\
& \leqq \log \operatorname{Tr}_{\mathscr{H}}\left(e^{-\beta_{0} H}\right)-\left(\beta-\beta_{0}\right) \hat{H}(\varrho)
\end{aligned}
$$

and thus the desired result follows with $m$ given by

$$
m=\left(\beta-\beta_{0}\right)^{-1}\left[\log \operatorname{Tr}_{\mathscr{H}}\left(e^{-\beta_{0} H}\right)-n\right] .
$$

To deduce the semi-continuity of the conditional entropy over $E_{\mathscr{O}}$ it suffices to show that each of the sets $D_{n}(S ; \beta H)$ is closed in the weak ${ }^{*}-\mathfrak{A}$ topology. But from the above argument it then suffices to show that $\omega_{e} \in D_{n}(H) \rightarrow S\left(\omega_{\varrho} ; \beta H\right)$ is upper semi-continuous for each $n$.

Now with the notation used in the proof of Theorem 4, we have for each $\omega_{\varrho} \in D_{n}(H)$

$$
S\left(\omega_{\varrho} ; \beta H\right)-\log \operatorname{Tr}_{\mathscr{H}}\left(e^{-\beta H}\right)=\sum_{m \geqq 1} x_{m}
$$

where $x_{m}$ is defined by

$$
\begin{aligned}
x_{m}= & -\lambda_{m}(\varrho) \log \lambda_{m}(\varrho)-\lambda_{m}(\varrho)\left[\beta\left\|H^{\frac{1}{2}} \Psi_{m}\right\|^{2}+\log \operatorname{Tr}_{\mathscr{H}}\left(e^{-\beta H}\right)\right] \\
& +\lambda_{m}(\varrho)-\frac{\left(\Psi_{m}, e^{-\beta H} \Psi_{m}\right)}{\operatorname{Tr}_{\mathscr{H}}\left(e^{-\beta H}\right)} .
\end{aligned}
$$

But for each $m$ we have

$$
\begin{aligned}
x_{m} & \leqq-\lambda_{m}(\varrho)\left[\log \lambda_{m}(\varrho)-\log \frac{\left(\Psi_{m}, e^{-\beta H} \Psi_{m}\right)}{\operatorname{Tr}_{\mathscr{H}}\left(e^{-\beta H}\right)}\right]+\lambda_{m}(\varrho)-\frac{\left(\Psi_{m}, e^{-\beta H} \Psi_{m}\right)}{\operatorname{Tr}_{\mathscr{H}}\left(e^{-\beta H}\right)} \\
& \leqq 0
\end{aligned}
$$

where we have successively applied the two inequalities used in the proof of Theorem 4. From this last inequality, we can. deduce that

$$
S\left(\omega_{\varrho} ; \beta H\right)-\log \operatorname{Tr}_{\mathscr{H}}\left(e^{-\beta H}\right)=\inf _{M} \sum_{m \leqq M} \mathrm{x}_{m} .
$$

Now by Theorem 3 the weak*-2Y topology and the uniform topology coincide on $D_{n}(H)$ and by Theorem 2, and the remark at the end of its proof, the eigenvalues and eigenprojectors of $\varrho$ are continuous, in this topology, over $D_{n}(H)$. Finally noting that one can write

$$
-\beta\left\|H^{\frac{1}{2}} \Psi_{m}\right\|^{2}=\inf _{N}-\left(\Psi_{m}, H_{N} \Psi_{m}\right),
$$


where $H_{N}$ is the truncated form of $H$ used in the definition of $\hat{H}$ in Section 3 , one sees that $\omega_{\varrho} \in D_{n}(H) \rightarrow S\left(\omega_{\varrho} ; \beta H\right)$ is the lower envelope of a family of functions which are continuous in the weak*-2I topology induced on $D_{n}(H)$. Thus the conditional entropy is upper semi-continuous in this topology over each $D_{n}(H)$ and consequently over $E_{\mathfrak{H}}$. We emphasize that the continuity properties of Theorem 5 are valid for each irreducible $C^{*}$-algebra $\mathfrak{A}$ on $\mathscr{H}$.

\section{Locally Normal States}

In mathematical physics one is often interested in a situation more general than the one we have discussed in the foregoing sections. In particular one is seldom interested in the normal states of $C^{*}$ algebras but in the locally normal states of algebras with a local structure. We will next consider this situation from a similar viewpoint to that adopted for normal states. We first introduce the notions of a locally normal state and an algebra with local structure. We will not attempt to give the most general definitions but consider a structure typical of that used to describe systems of bose particles, i.e. the structure typically related with the $C^{*}$ algebras associated with the canonical commutation relations.

First consider a collection of Hilbert spaces $\left\{\mathscr{H}_{A}\right\}$, where $A$ runs over the bounded open sets of $R^{v}$, which are interrelated by the tensor product property

$$
\mathscr{H}_{\Lambda_{1} \cup A_{2}}=\mathscr{H}_{\Lambda_{1}} \otimes \mathscr{H}_{A_{2}}
$$

whenever $\Lambda_{1} \cap A_{2}=\emptyset$. Next introduce a family $\left\{\varrho_{A}\right\}$ of compatible density matrices to be a family with the properties that $\varrho_{A}$ is a density matrix on $\mathscr{H}_{\Lambda}$, for each $\Lambda$, and

$$
\varrho_{A_{1}}=\operatorname{Tr}_{\mathscr{H}_{A_{2}}}\left(\varrho_{A_{1} \cup A_{2}}\right)
$$

whenever $\Lambda_{1} \cap \Lambda_{2}=\emptyset$. Next we assume that we have a collection of $C^{*}$ algebras $\left\{\mathfrak{H}_{A}\right\}$ which satisfy the following conditions:

1. For each $\Lambda, \mathfrak{H}_{A}$ is an irreducible $C^{*}$ algebra of operators on $\mathscr{H}_{\Lambda}$.

2. If $\Lambda_{1} \subset \Lambda_{2}$ then $\mathfrak{U}_{\Lambda_{1}} \subset \mathfrak{A}_{\Lambda_{2}}$. [In this second condition we implicitly identify $\mathfrak{H}_{\Lambda_{1}}$ on $\mathscr{H}_{\Lambda_{1}}$ with the algebra $\mathfrak{H}_{\Lambda_{1}} \otimes 1_{\Lambda_{2} \backslash \Lambda_{1}}$ on $\mathscr{H}_{\Lambda_{2}}$ where $1_{\Lambda_{2} \backslash \Lambda_{1}}$ is the identity operator on $\mathscr{H}_{\Lambda_{2} \backslash \Lambda_{1}}$.] Finally we define the $C^{*}$ algebra $\mathfrak{A}$ to be the uniform closure of all $\mathfrak{A}_{A}$.

In the sequel we will always assume that the families, $\left\{\mathscr{H}_{A}\right\}$ of Hilbert spaces, $\left\{\varrho_{A}\right\}$ of density matrices, and $\left\{\mathfrak{H}_{A}\right\}$ of $C^{*}$ algebras, under discussion satisfy the above conditions.

Now each family $\left\{\varrho_{A}\right\}$ of density matrices determines a state $\omega_{\varrho}$ over $\mathfrak{A}$ by the definition

$$
\omega_{\varrho}(A)=\operatorname{Tr}_{\mathscr{H}_{A}}\left(\varrho_{A} A\right)
$$


for each $A$ and each $A \in \mathfrak{A}_{A}$, and by continuous extension to the elements of $\mathfrak{A}$ which are not contained in some $\mathfrak{A}_{A}$. Conversely if we define $L$ to be the set of states $\omega$ over $\mathfrak{A}$ which have the property that the restriction of $\omega$ to each $\mathfrak{A}_{A}$ is normal then it is easily checked that $\omega$ defines a family of compatible density matrices. We call $L$ the set of locally normal states over $\mathfrak{A}$ and identify the families $\left\{\varrho_{A}\right\}$ of density matrices with the corresponding states $\omega_{\varrho} \in L$.

Now note that although the family $\left\{\mathscr{H}_{A}\right\}$ uniquely determines the set of all families $\left\{\varrho_{A}\right\}$ of compatible density matrices, it does not generally determine the $C^{*}$ algebra $\mathfrak{A}$ in a unique manner. Typically there are several different families $\left\{\mathfrak{H}_{A}\right\}$ of local $C^{*}$ algebras which satisfy the imposed properties and these families determine different $C^{*}$ algebras $\mathfrak{A}$. Thus the set $L$ of locally normal states can be associated with several different $C^{*}$ algebras $\mathfrak{A}$ and can be considered as the common component of the state spaces of all such algebras. We will again consider the set $L$ of locally normal states as the central object of our investigation and refer to each $C^{*}$ algebra $\mathfrak{A}$ with the above structure as an algebra associated with $L$.

The set $L$, or its subsets, can be equipped with many topologies. If $\mathfrak{A}$ is a $C^{*}$ algebra associated with $L$ we can equip this set with the weak ${ }^{*}-\mathfrak{A}$ topology, or alternatively, by restricting the states in $L$ to the subalgebras $\mathfrak{U}_{A}$ of $\mathfrak{A}$ we can equip the set with the weak ${ }^{*} \mathfrak{U}_{A}$ topologies or the uniform- $\mathfrak{H}_{A}$ topologies. We have seen in previous sections that there are many situations in which these latter topologies can coincide. But if we now consider subsets of $L$ on which the weak $*-\mathfrak{H}_{A}$ and uniform- $\mathfrak{H}_{A}$ topologies coincide for all $\Lambda$ then there are immediate implications for the weak*-2I topologies.

Theorem 6. Let $L$ be the set of locally normal states and let $\mathfrak{A}$ and $\mathbb{Q}$ be two $C^{*}$ algebras associated with $L$. Denote the generating families of $\mathfrak{A}$ and $\mathfrak{Q}$ by $\left\{\mathfrak{Q}_{A}\right\}$ and $\left\{\mathfrak{I}_{A}\right\}$. Let $K$ be a subset of $L$ with the property that the weak ${ }^{*}-\mathfrak{Q I}_{A}$ and weak ${ }^{*}-\mathfrak{I}_{A}$ topologies coincide on $K$ for each $\Lambda \subset R^{*}$.

It follows that the weak ${ }^{*}-\mathfrak{I}$ and weak ${ }^{*}-\mathfrak{Q}$ topologies coincide on $K$.

Assume further that the weak ${ }^{*}-\mathfrak{U}_{A}$ and the uniform- $\mathfrak{U}_{A}$ topologies coincide on $K$ for each $\Lambda \subset R^{v}$ then it follows that $K$ is metrizable in the weak*-2l topology.

Proof. The first statement of the theorem is a trivial consequence of the definition of $\mathfrak{A}$ and $\mathfrak{L}$ as the uniform closures of the families $\left\{\mathfrak{U}_{A}\right\}$ and $\left\{\mathbb{Q}_{1}\right\}$ respectively.

The second statement results from the facts that the uniform- $\mathfrak{H}_{A}$ topologies are metric topologies and that $R^{v}$ is metrizable. In fact a metric can be constructed for the weak*-2I topology as follows. Let $\Lambda_{n}, n=1,2, \ldots$ denote balls of radius $n$ centred at the origin and introduce 
the definition

Note that

$$
\left\|\omega_{\varrho}-\omega_{\sigma}\right\|_{n}=\sup _{A \in \mathscr{U}_{A_{n}}\|A\|=1}\left|\omega_{\varrho}(A)-\omega_{\sigma}(A)\right|, \quad \omega_{\varrho}, \omega_{\sigma} \in K .
$$

$$
\left\|\omega_{\varrho}-\omega_{\sigma}\right\|_{1} \leqq\left\|\omega_{\varrho}-\omega_{\sigma}\right\|_{2} \leqq \cdots .
$$

Now by the assumption that the weak $*-\mathfrak{A}_{A}$ and uniform- $\mathfrak{A}_{A}$ topologies coincide, we can deduce that the neighbourhoods of each $\omega_{Q} \in K$, defined by

$$
\mathscr{W}\left(\omega_{\varrho} ; n, \varepsilon\right)=\left\{\omega_{\sigma} ; \omega_{\sigma} \in K,\left\|\omega_{\varrho}-\omega_{\sigma}\right\|_{n}<\varepsilon\right\}
$$

form a basis for the weak*-2 topology induced on $K$. It then follows from a standard argument (see, for example, [4], pages 208-209) that the weak ${ }^{*}-\mathfrak{A}$ topology on $K$ is determined by the following metric

$$
\left\|\omega_{\varrho}-\omega_{\sigma}\right\|=\sum_{n \geqq 1} \frac{1}{2^{n}} \frac{\left\|\omega_{\varrho}-\omega_{\sigma}\right\|_{n}}{1+\left\|\omega_{\varrho}-\omega_{\sigma}\right\|_{n}} .
$$

The above theorem is principally of interest in combination with the results of the previous sections, for example, in combination with Theorems 3 and 5.

Finally we generalize a theorem, essentially due to Powers [2], concerning the characterization of locally normal factor states. For this purpose it is useful to introduce the algebra $\tilde{\mathfrak{U}}_{A}$ (cf. [5]) to be the $C^{*}$ subalgebra of $\mathfrak{A}$ generated by $\left\{\mathfrak{U}_{\Lambda^{\prime}} ; \Lambda^{\prime} \cap \Lambda=\emptyset\right\}$. Note that the definition of the subalgebras $\mathfrak{U}_{A}$ of $\mathfrak{U}$ is such that

$$
\left[\mathfrak{Q}_{\Lambda_{1}}, \mathfrak{U}_{\Lambda_{2}}\right]=0
$$

whenever $\Lambda_{1} \cap \Lambda_{2}=\emptyset$ and consequently one has

$$
\left[\tilde{\mathfrak{U}}_{\Lambda}, \mathfrak{U}_{\Lambda}\right]=0 \text { for all } \Lambda \subset R^{v} \text {. }
$$

Theorem 7. Let $\mathfrak{A}$ and $\mathfrak{Q}$ be two $C^{*}$ algebras associated with the set $L$ of locally normal states and denote the generating families of $\mathfrak{U}$ and $\mathfrak{Q} b y$ $\left\{\mathfrak{U}_{A}\right\}$ and $\left\{\mathfrak{Q}_{A}\right\}$. For each $\omega \in L$ the following four conditions are equivalent

$1_{\mathfrak{A}}\left(1_{\mathfrak{g}}\right) . \omega$ is a factor state of $\mathfrak{U}$ (of $\mathfrak{Z}$ ).

$2_{\mathfrak{Q}}\left(2_{\mathfrak{Q}}\right)$. Given $\Lambda \subset R^{v}$ and $\varepsilon>0$ there is a $\Lambda_{0} \subset R^{v}$ such that

$$
|\omega(A B)-\omega(A) \omega(B)|<\varepsilon\|A\|\|B\|
$$

for all $A \in \mathfrak{Q}_{\Lambda}\left(\right.$ all $\left.A \in \mathfrak{Q}_{\Lambda}\right)$ and all $B \in \tilde{\mathfrak{U}}_{\Lambda_{0}}\left(\right.$ all $\left.B \in \tilde{\mathfrak{Q}}_{\Lambda_{0}}\right)$.

This theorem was implicitly proved by Powers [2] in a special case where the underlying Hilbert spaces are finite dimensional. In this case there is a unique $C^{*}$ algebra $\mathfrak{U}$ associated with the corresponding set 
of locally normal states and as each $\mathfrak{U}_{A}$ is finitely generated the double uniformity of condition $2_{2}$ is clearly equivalent to uniformity in the "outer" algebra $\tilde{\mathfrak{I}}_{A_{0}}$. The proof of the simplified theorem, i.e. the characterization of factor states of $\mathfrak{A}$ by a singly uniform clustering condition, is then provided by Powers' estimates concerning families of normally embedded Type- $I_{n}$ factors. In the more general case under consideration various authors realized that Powers' estimates could be extended to families of normally embedded Type- $I_{\infty}$ factors ${ }^{3}$ and have consequently given characterizations of locally normal factor states by a cluster property which is uniform in the "outer" algebra $\tilde{\mathfrak{A}}_{\Lambda_{0}}[5,6]$. It has however been overlooked that the local normality condition rather trivially implies uniformity in the "inner" algebra $\mathfrak{P}_{A}$; this is established by an estimate of the type made in Sections 2 and 3.

We will not give a detailed proof of the theorem but simply list the main points of such a proof. It suffices to establish the equivalences for a general algebra $\mathfrak{U}$ associated with $L$ and the special algebra $\mathfrak{I}$ defined by the family of subalgebras $\mathfrak{Q}_{A}=\mathfrak{Q}\left(\mathscr{H}_{A}\right)$. But condition $2_{\mathfrak{Q}}$ then trivially implies condition $2_{\mathfrak{I}}$ because $\mathfrak{A}_{A} \subset \mathfrak{I}_{A}$ etc. Let $\pi(\mathfrak{U})$ and $\pi(\mathfrak{L})$ denote the representations of $\mathfrak{A}$ and $\mathfrak{Q}$ constructed in the canonical fashion from $\omega$. The irreducibility of the $\mathfrak{A}_{A}$ on $\mathscr{H}_{A}$ and the local normality of $\omega$ imply easily that the strong closures of $\pi\left(\mathfrak{U}_{A}\right)$ and $\pi\left(\mathfrak{L}_{A}\right)(\pi(\mathfrak{H})$ and $\pi(\mathfrak{L}))$ are identical and hence the corresponding centres $z_{\mathfrak{Q}}$ and $z_{\mathfrak{I}}$ are identical. Thus conditions $1_{\mathscr{Q}}$ and $1_{\mathfrak{\Omega}}$ are equivalent. The arguments of $[2,5,6]$ show that

$$
z_{\mathfrak{Q}}=z_{\mathfrak{L}}=\bigcap_{A \subset R^{v}} \pi\left(\tilde{\mathfrak{U}}_{A}\right)^{\prime \prime}=\bigcap_{\Lambda \subset R^{v}} \pi\left(\tilde{\mathfrak{I}}_{A}\right)^{\prime \prime}
$$

and applying this result in the manner of $[2,5,6]$ one finds that $2_{\mathscr{A}}$ implies $1_{\mathscr{R}}$ and $1_{\mathfrak{Q}}$ implies that if $\varepsilon>0$ and $E$, a finite rank projector in $\mathfrak{Q}_{\Lambda}$, are given then there is a $\Lambda_{0} \supset \Lambda$ such that

$$
|\omega(E A E B)-\omega(E A E) \omega(B)|<\frac{\varepsilon}{2}\|A\|\|B\|
$$

for all $A \in \mathfrak{Q}_{A}$ and all $B \in \tilde{\mathfrak{I}}_{A_{0}}$. But given $\varepsilon>0$ we can choose $E \in \mathfrak{I}_{A}$ such that

$$
0 \leqq \omega(1-E)<\varepsilon / 12 \text {. }
$$

Here we use the fact that $\omega$ is normal in restriction to $\mathscr{Q}_{A}$. Finally we have

$$
\begin{aligned}
|\omega(A B)-\omega(A) \omega(B)| & <\frac{\varepsilon}{2}\|A\|\|B\|+|\omega(E A E B)-\omega(E A E) \omega(B)| \\
& <\varepsilon\|A\|\|B\|
\end{aligned}
$$

for all $A \in \mathfrak{Q}_{A}$ and $B \in \tilde{\mathfrak{I}}_{\Lambda_{0}}$. Thus $1_{\mathfrak{I}} \Rightarrow 2_{\mathfrak{I}} \Rightarrow 2_{\mathfrak{I}} \Rightarrow 1_{\mathfrak{R}} \Rightarrow 1_{\mathfrak{I}}$ and the proof is complete.

${ }^{3}$ This was first communicated to the author by O. E. Lanford in June 1968.

17 Commun math Phys., Vol 19 
Acknowledgements. The author is indebted to H. Borchers for a number of useful suggestions concerning the material contained in Section 2. A number of informative discussions were held with S. Miracle-Sole and D. Kastler. We are also grateful to G. ZellerMeier for pointing out a number of errors in an earlier version of this work.

\section{References}

1. Miracle-Sole, S., Robinson, D. W.: Commun. Math. Phys. 14, 235 (1969).

2. Powers, R.: Ann. Math. 86, 138 (1967).

3. Riesz, F., Nagy, B. Sz.: Leçons d'analyse fonctıonnelle. Paris: Gauthier-Villars 1965.

4. Köthe, G.: Topologische lineare Räume. Berlin-Heidelberg-New York: Springer 1966.

5. Lanford, O. E., Ruelle, D.: Commun. Math. Phys. 13, 194 (1969).

6. Haag, R., Kadison, R., Kastler, D.: Commun. Math. Phys. 16, 81 (1970).

D. W. Robinson

Centre de Physique Théorique - C.N.R.S.

31, Chemin Joseph Aiguier

F-13 Marseille 9e 